\title{
Labyrinthe
}

21 | 2005 (2)

Communauté en pièces : d'Europe, d'Islam et d'ailleurs

\section{Enjeux de nomination}

Les usages savants de la communauté

\section{Élise Massicard}

\section{(2) OpenEdition}

\section{Journals}

Édition électronique

URL : http://journals.openedition.org/labyrinthe/902

DOI : $10.4000 /$ labyrinthe.902

ISSN : 1950-6031

Éditeur

Hermann

Édition imprimée

Date de publication : 22 juin 2005

Pagination : 49-52

\section{Référence électronique}

Élise Massicard, «Enjeux de nomination », Labyrinthe [En ligne], 21 | 2005 (2), mis en ligne le 10 juillet 2008, consulté le 20 avril 2019. URL : http://journals.openedition.org/labyrinthe/902 ; DOI : 10.4000/ labyrinthe.902

Propriété intellectuelle 


\title{
ENJEUX DE NOMINATION \\ Les usages savants de la communauté
}

\author{
Élise MASSICARD
}

Comment comprendre l'emploi souvent spontané1, y compris par les chercheurs occidentaux, du terme «communauté alévie ${ }^{2}$ » pour désigner ce groupe en Turquie comme en migration, alors même que cette qualification semble loin d'être une évidence scientifique ? $\leadsto$ « La communauté, un universel sociologique ?» $»$ Pourquoi le rapport 2003 de la Commission européenne qualifie-t-il les alévis de «communauté musulmane non sunnite», celui de 2004 de «communauté religieuse $^{3} »$ ? Bien que beaucoup d'auteurs s'accordent à reconnaître le caractère construit ou «imaginé» de la «communauté alévie ${ }^{4}$ », celleci est ensuite souvent traitée comme un donné et un tout monolithique. Enfin et surtout, comment expliquer que cette qualification semble si peu faire l'objet de débats?

1. Notons la démarche de clarification, encore trop rare, adoptée à ce sujet par Hamit Bozarslan: «L'alévisme et l'impossible équation du nationalisme en Turquie », dans Riva Kastoryano, Alain Dieckhoff (dir.), Nationalismes en mutation en Méditerranée orientale, Paris, éditions du CNRS, 2002.

2. Par exemple : «On peut espérer [pour les alévis] et leur communauté que la voie de l'émancipation dans laquelle ils se sont engagés soit une voie de la paix, du respect et de la tolérance », Karin Vorhoff, Zwischen Glaube, Nation und neuer Gemeinschaft : alevitische Identität in der Türkei der Gegenwart, Berlin, Klaus Schwarz, 1995, p. 5. Voir aussi Ragnar Naess, « Being an Alevi Muslim in South-Western Anatolia and in Norway : The Impact of Migration on a Heterodox Turkish Community », dans Tomas Gerholm, Yngve G. Lithman (dir.), The New Islamic Presence in Western Europe, Londres, Mansell, 1988, p. 174-195 ; Hamit Bozarslan, « L'alévisme, la méta-histoire et les mythes fondateurs de la recherche», dans Isabelle Rigoni (dir.), Turquie : les mille visages. Politique, religion, femmes, immigration, Paris, Syllepse, 2000.

3. Commission des Communautés européennes, Rapport régulier 2003 sur les progrès réalisés par la Turquie sur la voie de l'adhésion, Bruxelles, 5 novembre 2003, URL :

http://europa.eu.int/comm/enlargement/report_2003/pdf/rr_tk_final_fr.pdf

4. Benedict Anderson, Imagined Communities : Reflections on the Origin and Spread of Nationalism, Londres, Verso, 1983, trad. fr., L'Imaginaire national : réflexions sur l'origine et l' essor du nationalisme, Paris, La Découverte, 1996. Voir notamment Karin Vorhoff, «"Let's Reclaim our History and Culture !" - Imagining Alevi Community in Contemporary Turkey », Die Welt des Islams, 1998, vol. 38 (2), p. 220-252 ; Martin Sökefeld, «Alevism Online : Re-Imagining a Community in Virtual Space », Diaspora - A Journal of Transnational Studies, 11, 2002, p. 85-123. 
On pourrait déceler ici l'héritage (assumé ou non) d'une tradition orientaliste, qui imprégnerait les perceptions savantes des alévis. Mais une telle affirmation apparaît simplificatrice. En effet, les alévis ont été très peu étudiés durant l'âge d'or de l'orientalisme; en outre, ils n'ont guère été mêlés à l'impérialisme occidental lié à l'émergence et à la consolidation de la tradition orientaliste. Enfin, la Turquie républicaine est l'un des pays de la région les moins marqués par cette grille de lecture en termes de «communautés», à la différence des Balkans (exYougoslavie) ou du Moyen-Orient (Liban, mais aussi Syrie; Irak par exemple). Elle est au contraire largement abordée à travers les problématiques de la modernisation, du changement social, problématiques dans lesquelles les sous-groupes sont peu étudiés en tant que tels. Ainsi, si beaucoup d'historiographies consacrées à d'autres pays de la région s'appuient sur l'idée d'une continuité entre l'héritage ottoman et ce qui a suivi, notamment concernant les « communautés», cela est probablement moins le cas concernant la Turquie. $\rightarrow$ « La communauté d'historicité... »

\section{Orientalisme}

La tradition orientaliste tend à décoder la diversité humaine de l'« Orient compliqué " avant tout à travers le fait religieux, considéré comme ayant une importance fondamentale et dont découleraient les autres dimensions de la vie sociale ${ }^{5}$. De ce fait, les unités sociales pertinentes y sont des groupes avant tout religieux, souvent vus comme groupes englobants. Ces sociétés " orientales " sont généralement considérées comme passéistes, traditionalistes, en quelque sorte en retard sur les sociétés occidentales ; leur dimension " communautaire " - au sens péjoratif du terme, au moins en français - implique que « l'émergence de l'individu » est largement appréhendée comme un processus problématique.

Pourquoi cette différence? Tout d'abord, les études sur la Turquie contemporaine sont marquées par le présupposé d'une rupture radicale qu'auraient introduite la « révolution » et la fondation de la République sur les cendres de l'Empire, rupture qui forme la base de l'identité

5. Edward Said, Orientalism, New York, Pantheon, 1978 ; trad. fr. C. Malamoud, L'Orientalisme, l'Orient créé par l'Occident, Paris, Seuil, 1980. 


\section{Enjeux de nomination - Les usages savants de la communauté}

nationale turque. En outre, la moindre prégnance de la grille de lecture «communautaire» s'explique probablement en partie par l'idéologie officielle unitariste de la Turquie, pour laquelle tout particularisme est illégitime. On peut cependant émettre l'hypothèse que, dans ces qualifications savantes des alévis de «communauté», on trouve l'influence d'une tradition de recherche plus large et plus ancrée, ce qui renvoie à la question de la structuration des champs de recherche en Europe ${ }^{6}$.

Face à cette situation, il nous semble plus prudent de ne pas qualifier les alévis dans leur ensemble de "communauté». Si cela a pu correspondre dans une certaine mesure à la situation à une certaine époque de l'Empire ottoman (la période de consolidation du groupe Klzılba - autour de la référence safavide, au $\mathrm{XVI}^{\mathrm{e}}$ siècle) - ce qui reste à démontrer -, cette qualification n'est qu'imparfaitement applicable aux alévis contemporains, tout d'abord parce qu'ils ne sont pas organisés autour de la religion, mais aussi en raison de la coexistence de nombreux sous-groupes diversifiés et de la faible organisation du groupe. Pour les mêmes raisons, je parle d'«alévis» et non pas d' «Alévis ». Le problème de la majuscule est fondamental, car lié à la nature de l'alévité, à la fois religion, culture, appartenance à un groupe humain, qui est à la fois plus et moins qu'un groupe religieux. Il s'agit d'un choix idéologique, puisqu'il implique une décision sur la nature de l'alévité, confession ou peuple. Or, ce choix m'apparaît impossible, puisque la nature ambiguë de l'alévité constitue, pour moi, l'une des variables explicatives essentielles (ambiguïté que l'on retrouve au sujet des Turcs de Thrace $\rightarrow$ «Minorité/communauté : la cemaat... » $\nleftarrow)^{7}$. Je m'en tiens donc à la minuscule, pour la raison simple qu'elle fige moins la réalité. Cette perspective ne doit cependant pas conduire à un relativisme outrancier : la volonté de comprendre les conditions de production des discours relatifs à l'alévité ne peut en aucun cas conduire à nier l'existence de celle-ci.

Il s'agit donc de renverser le problème: plutôt que de prendre le groupe alévi comme un «tout» et un «donné», il s'agit de mettre en

\footnotetext{
6. «La notion même d'un domaine de recherches fondé sur une unité géographique, culturelle, linguistique et ethnique appelée l'Orient», Edward Said, op. cit., p. 66. Beaucoup de scientifiques ayant étudié les alévis sont des spécialistes de la région (ottomanistes, turcologues, islamologues...) ou s'inscrivent dans ces champs d'études.

7. Voir, pour le même problème par rapport au judaïsme, Dominique Schnapper, Juifs et israélites, Paris, Gallimard, 1980, p. 34
} 


\section{Labyrinthe, $n^{\circ} 21$}

avant son caractère problématique et de comprendre pourquoi et comment, dans ces conditions, peut être revendiquée une communauté. Par rapport à la majorité des études sur les alévis, cette analyse se déplace de l'architecture et de l'histoire des unités sociales et des formes symboliques vers celle des dynamiques historiques et politiques de leur production et de leur reproduction. Cette ambition se heurte à une interrogation sur la validité de l'objet: est-il pertinent d'écrire l'histoire sociale d'un groupe dont la cohérence et l'existence même ne vont pas de soi ? Loin de politiser l'alévité, cette approche doit au contraire dévoiler les ressorts politiques des discours et mobilisations autour de cette notion.

Une mise en garde se révèle donc nécessaire contre un usage spontané et non réflexif de la «communauté», usage qui nous semble particulièrement répandu au sujet de groupes d'origine religieuse dans (ou originaires) des régions comme le Proche-Orient. Postuler d'emblée un caractère «communautaire» induit le danger de masquer la diversité des types d'organisation sociale sous une homogénéité de façade réductrice (la «communauté juive» de France est-elle vraiment organisée comme la «communauté musulmane» ou la «communauté alévie»?), de masquer l'absence d'organisation sociale spécifique ou de cohésion d'un groupe, voire de réifier le groupe analysé. Définir la communauté en fonction de concepts préétablis par les chercheurs peut de ce fait brouiller plus qu'éclairer le fonctionnement réel des phénomènes sociaux. De ce fait, le terme, plus neutre, de «groupe», semble préférable, sans qu'en soient qualifiés plus avant la nature, le fonctionnement et la cohésion. Le phénomène peut alors être examiné sans imposer de l'extérieur un modèle porteur d'implications politiques. 